\title{
Hard, Harder, and the Hardest Problem: The Society of Cognitive Selves
}

\author{
Venkata Rayudu Posina*
}

\begin{abstract}
The hard problem of consciousness is explicating how moving matter becomes thinking matter. Harder yet is the problem of spelling out the mutual determinations of individual experiences and the experiencing self. Determining how the collective social consciousness influences and is influenced by the individual selves constituting the society is the hardest problem. Drawing parallels between individual cognition and the collective knowing of mathematical science, here we present a conceptualization of the cognitive dimension of the self. Our abstraction of the relations between the physical world, biological brain, mind, intuition, consciousness, cognitive self, and the society can facilitate the construction of the conceptual repertoire required for an explicit science of the self within human society.
\end{abstract}

Keywords: Category Theory, Consciousness and Mind, Monad Self

\section{Introduction}

The nature of the experiential self is a highly debated topic in philosophy. Various schools of thought have been arguing that there is no self. These arguments against the reality of the self are usually based on analogies such as 'row of ants' (Siderits, Thompson, \& Zahavi, 2011, pp. 1-26) and 'center of gravity' (Dennett, 1992). Countering these analogy-based mischaracterizations of the self as an illusion, fiction, or myth (cf.

* NIAS-Consciousness Studies Programme, Bangalore, India; posinavrayudu@gmail.com 
Metzinger, 2009), Menon (2014) argued for recognizing the self, which is given in subjective experiences, as the experiencer (see also Midgley, 2014). Here we bring out the conceptual necessity of the self by way of extending the recent idealization of ordinary cognition as mathematical knowing (Posina, Ghista, and Roy, 2017), which is based on Lawvere's (1994a) 'Categorical Refinement of a Hegelian Principle' (see also Lawvere, 2013a). The basis of our arguments is the representational character of conscious experiences (Chalmers, 2006). Simply stated, conscious experiences are representations of some state-of-affairs of the world, and thanks to these subjective experiences the experiencing subject gets to know about the world. Upon realizing that we have a definite understanding of the notion of 'representation' within the collective mathematical practices of representing reality (Lawvere, 1963; Lawvere, 1994a; Lawvere, 2004; Lawvere, 2013b), the author examined mathematical experience to discern the implications of the representational character of subjective experiences. With conscious experiences as subjective representations of objective things, we find that, going by the mathematical experience, there must be analogues of measured properties, abstract theories, backgrounds (for interpreting theories to obtain representations), and mathematical doctrines, which make subjective experiences representations of one or another thing. Upon comparing neural coding to measurement of properties, mental concepts to abstract theories, intuition to background, and the self to a doctrine, which determines how a given state-of-affairs is represented, we characterize the decisive abstract general relations between the material world, biological brain, mind, intuition, consciousness, and the self within human society.

One could begin with Hume, who pointed out that, when one looks into oneself, one finds subjective experiences such as the sight of a sunset, or the sound of a bell, or the smell of a rose; and that is all there is (Hume, 1978). More than anything else, what Hume's experience tells us is that the method that detects subjective experiences does not detect the subject. Based on this observation we can conclude that subjective experiences and the experiencing subject are two different kinds of entities. Introspective exercises such as these, nevertheless, led many to conclude that there is no self or that the self is at most a bundle of subjective experiences. 76 
Even within this bundle theory of self, self is an entity distinguishable from subjective experiences by virtue of its distinct structure and properties vis-à-vis those of subjective experiences. More specifically, the self as a bundle [of subjective experiences] has the property of size (e.g. big bundle, small bundle), which subjective experiences themselves do not have. In more formal terms, a set [of elements] is a distinct structure distinguishable from the elements that the set contains (Lawvere, 1994b; Lawvere \& Rosebrugh 2003, p. 1). To deny the existence of the self [on the grounds that it is no more than a bundle of subjective experiences] is, from this perspective, as sensible as denying the existence of sets [as distinct from the elements that they contain]. A related argument against enduring selves is based on the idea that there is no subject without subjective experiences (Strawson, 2009). With subjective experiences as elements and the self as a set formed of the subjective experiences, we find that there can be a self without subjective experiences. If we remove all the elements from any set, then what we have is a set-an empty set-and not a situation of 'no set'. In other words, with the self as a totality of subjective experiences (irrespective of how one formalizes totality), selves exist with or without subjective experiences, which highlights the inconceivability of the claim: "no experience, no subject" (Gallagher 2011, p. 9). The reality of the self, as the subject given in subjective experiences, can be made more palpable by noting the conceptual kinship between conscious experience and description, as follows.

Consider a commonplace experience: seeing a cursor blinking on a computer screen. Where is the self in this conscious experience of seeing a blinking cursor? Is the self an artifact of articulation brought into being when describing one's experiences as in: "I see a blinking cursor"? More pointedly, is there anything to this ' $\mathrm{I}$ ' above and beyond the sights and sounds to which it is prefixed (cf. I see..., I hear...)? Yes, the self is intrinsic to and yet distinct from conscious experience, which can be readily seen by comparing conscious experiences to descriptions. A sentence such as 'Rama killed Ravana' readily signifies an event of which it is a description. Though not as readily, it also exemplifies the grammar, i.e., subjectverb-object structure underlying that particular description. Along similar lines, an equation: ' $y=x$ ' readily signifies a straight line; and that signification is made possible by Cartesian geometry 
(Lawvere \& Schanuel, 2009, pp. 41-42). These illustrations indicate that the processes involved in describing are not as readily discernible [as that which is described] in the descriptions. But, that is no license to deny either the grammar of verbal descriptions or the mathematics of mathematical modeling. So is the case with the self of subjective experiences. Just as grammar determines what can be said and what must be said in describing any given state-of-affairs, and just as mathematics determines how to model any given situation, so does the self: one's self determines how one experiences. Encouraged by these preliminary clarifications, we embarked on a thorough investigation of the parallels between mathematics and cognition.

Mathematics is about knowing and so is the self (Menon, 2014, p. 1). In drawing parallels between individual cognition and collective mathematical knowing, we realized the indispensability of the notion of self (as discussed in the section titled 'The Knowing Self'). Our immediate objective here is to clarify various notions of the consciousness studies in terms of better-understood constructs drawn from the domain of mathematics. The present paper documents the feasibility and fruitfulness of this research program by way of identifying analogues of the self, consciousness, mind, brain, and the world within the practice of mathematical knowing. The mathematical analogues, in turn, suggest a formalization of the relations between the material world of things, their neural coding in the brain, mental concepts, conscious experiences, and the experiencing subject. Before we present our mathematical framework, we provide a brief account of the rationale behind our approach to the development of an explicit science of the self.

\section{On the Development of the Science of the Self}

How does one develop the science of the self? In broad terms, science is the alignment of reason with experience. Advancement of the science involves, additionally, the development of novel concepts that fit reality (given in planned perception or experimental findings). In reasoning about a given experience, oftentimes, the inadequacy of available concepts becomes clear, which fuels the search for simpler instances (exemplifying the essence of given experience), where abstraction of appropriate 
general concepts is relatively easy. Thus developed conceptual repertoire can then be brought to bear on comprehending the original motivating experiences. As an illustration, consider the study of motion. Motion, as is the case with any other experience, did not come equipped with the science of motion. And, of course, watching and wondering about the many ways things move: swimming fish, sprinting athletes, slithering snakes, shooting stars, sailing ships, racing thoughts, gushing winds, flying birds, flowing rivers, falling coconuts, drifting clouds, changing seasons, buzzing bees, beating hearts, etc. did not result in Newton's universal laws of motion. A key step in the development of the science of motion is Galileo's study of the motion of a falling stone, which might have seemed too simplistic to inform our understanding of the more fascinating, say, flight of the birds (Lawvere \& Schanuel, 2009, pp. $3-9,308-309)$. However, it is this skeletal motion of dropped objects falling straight to the ground (and not plucking one feather after another of a bird to see its effect on the flight of the bird) that gave us the foundational concept of acceleration (to name one), which formed the basis for the subsequent development of more complicated concepts such as torque needed to provide a satisfactory scientific account of the bird flight, not to mention the applications of the science of motion leading all the way up to orbiting satellites, among other fantastical moving things.

Drawing parallels to the development of the science of motion, as in the case of motion, there are also many selves: spiritual self, social self, physical self, narrative self, mental self, experiential self, emotional self, embodied self, ecological self, conceptual self, and cognitive self, just to name a few (Gallagher, 2000). Paralleling the puzzlement over the very possibility of motion (cf. Zeno's paradox), we also have strange arguments against the very existence of the self (see 'Introduction'), all of which is reminiscent of a pre-Galilean state-of-affairs. Having recognized that the study of the self is in a pre-Galilean state, as a first step towards the development of the science of the self, we identified 'cognitive self' as the "falling body" of the science of the self in the sense 'knowing' exemplifies the essence of the self (see the section titled 'The Knowing Self'). 
The study of the self, not unlike the study of motion that necessarily involves space and time, is necessarily a study of the triangular relationship between objective reality, subjective experiences, and the experiencing subject. With knowing as an integral part of experiencing, we look for a model system to study the mutual relations between objects, subjective knowledge (resulting from thinking and seeing), and the knowing self. In particular, we would like to thoroughly characterize the determination of subjective experiences by both the subject and the object. Informally speaking, we are seeking a science of viewpoint, which determines how things appear. Upon focusing on individual cognition, we find that the notion of mathematical doctrine (also known as monad), which determines the generalization (theory and model) involved in knowing about a particular category of mathematical objects (Lawvere,1994a, Lawvere, 2004; Lawvere, 2013b), serves as an elementary form of the knowing self (determining the conception and perception of given stimuli). The functorial calculus of generalization (with respect to a doctrine) of the given particulars can facilitate the development of the science of the knowing self the way calculus facilitated the development of physics (Lawvere, 1999, p. 412). In the following, we motivate our conceptualization of the knowing self by elaborating on the added difficulty of accounting for the self, beyond that of mind and conscious experiences (Menon, 2014).

\section{The Harder Problem}

There are things in the world and we, the thinkers, think about things. Things are made up of matter and so are thinkers. Within this materialist world-picture, there are two kinds of things: (i) moving things and (ii) thinking things (Lawvere, 1980). What is not clear, in our scientific world-view, is how thinking things emerge from moving things. This is the largely acknowledged 'hard problem' of consciousness studies (Chalmers, 1995). Though not as widely acknowledged, the problem of transforming experiences into experiencing self is harder than the hard problem of transforming things into experience of things (Menon, 2014, pp. $2,34,50-60)$. The added difficulty is the inconceivability of 'perspective' emerging from observations. Perspective is a 
precondition for observing; observations are invariably from here, there, or somewhere (Campos \& Gutiérrez, 2015; Sen, 1993). Consider, for example, a cylinder standing on its base. The appearance of the cylinder depends on the viewpoint from which it is viewed. The cylinder appears as a circle, when viewed from top, and it appears as a rectangle when viewed from front. Note that it is the viewpoint 'top' that makes the circle an appearance of the cylinder. Appearance [of a thing] is an appearance [of the thing] from a perspective. The notion of 'perspective' cannot be reduced to observations; it is a conceptual prior that makes observation possible, which is along the lines of Kantian resolution of the conflicting-Descartes vs. Hume-claims regarding the reality of the self (Hamilton, 2015; Praetorius, 2016). Put differently, conscious experiences are about something; they represent some state-of-affairs (Chalmers, 2006). Conscious experiences, not unlike representations, are representative of that which they represent, but only from a certain perspective. A subjective experience that represents a certain state-of-affairs is representative of that state-ofaffairs only for the subject to whom the subjective experience belongs to. This is a simple consequence of the representational character of experience. For example, computer signifies a thing to me, but to someone who does not know English, the word "computer" is merely black contours on a white background. In other words, descriptions are descriptions of that which they describe, but only from a specific perspective (which in this case is a particular language). Upon admitting conscious experience, into reality, as an experience about one or another thing, as a reflective part of reality, we readily recognize conscious experience as an experience of a subject, which, in turn, forces us to admit the experiencing self, as a viewpoint making appearance possible, also into our conception of reality. This conception of reality consists of not only objects and observations (subjective appearances of objective things), but also observers (viewpoints). Reality, for the most part, has been analyzed in terms of two categories: Being (objectifying 'unity' as the category of reflexive graphs) and Becoming (objectifying 'change' as the category of dynamical systems; Lawvere, 1991; Lawvere, 1992; Lawvere, 1999). In other words, we attribute reality to things and their transformations. However, reality also consists of a special type of things such as 
books, theories, and brains that are reflective of the reality (Lawvere \& Schanuel, 2009, pp. 84-85; see also Sen, 1993). More importantly, reality includes perspectives, which personify the possibility of reflection i.e. the appearances of things. This notion of perspective, just like the self, cannot be reduced to objective things or subjective appearances (see Campos \& Gutiérrez, 2015). However, the relationship between subjective experiences, experiencing subjects, and objects of experience can be systematically investigated, as discussed below, in terms of the analogous situation of mathematical knowing involving generalization (theories and models) of objective particulars based on a doctrine.

Our subjective experiences (mental concepts, conscious percepts) are reflective of the objective reality. Based on these subjective reflections we make our way through the world. When viewed from the perspective of reflecting, the functorial calculus of mathematical knowing involving particulars, properties, theories, models, presentations, and doctrines provides a coherent framework to unify all six categories of consciousness studies: 1. Physics (stimuli), 2. Biology (neural codes), 3. Mind (concepts), 4. Consciousness (percept), 5. Language (descriptions), and 6. Self (locus of coding, thinking, seeing, and speaking). Our plan is to objectify the concept of REFLECTING as a Category of Reflecting (along the lines of the objectification of Being and Becoming as categories of reflexive graphs and dynamical systems, respectively). More specifically, since theories are reflective subcategories defined in terms of adjoint functors, the act of reflecting can be objectified as a category with monads of theorymodel adjoint functors as objects.

\section{The Knowing Self}

Self, according to the Indian philosophical text Bhagavad Git $\bar{a}$, is the knower (Menon, 2014, p. 1). Self is not merely the subject of one's subjective experiences; it is the knowing subject, which is selfaware. With 'self as self-aware knower', the self is required to include not only knowledge but also a declarative understanding of the procedural knowledge of acquiring knowledge. The selfconceptualized as a reflexive knower-is thus the higher-order 82 
knowledge of both knowing and knowledge. Given the definiteness of scientific knowledge, modeling 'self as scientist' can be a fruitful exercise leading to added insights into the nature of the self. Unfortunately, there is no scientific account, within science, of a scientist. Thankfully, within mathematical sciences, we have the mathematics of category theory, which not only accounts for mathematical knowledge but also for the processes of acquiring mathematical knowledge. Development of the science of knowing, according to F. William Lawvere, 'will require the use of the mathematical theory of categories. Even within mathematical experience, only that theory has approximated a particular model of the general, sufficient as a foundation for a general account of all particulars' (Lawvere, 1994a, p. 43). Thus, with regard to the reflexive-knowing attribute of the self, mathematics in general and, more specifically, the category of categories (Lawvere, 1966) constitutes a good model system to investigate self-awareness. A self-aware system is, minimally, one with a representation of itself within itself, i.e., there is a part within a whole which is reflective of the whole (or other parts of the whole). The category of categories is autonomous i.e. every category contains a discrete subcategory which is reflective of the category in the sense objects of a category can be represented within itself i.e. as diagrams in the discrete subcategory (Lawvere, 2004, pp. 11-12). Of course, the knowledge constituting the self is much more elaborate than mathematical knowledge. In fact, our arguments for the reality of the self are based on recognizing mathematical knowing as a special case of the more general individual cognition (Lawvere,1994a), and as an empirical phenomenon (Hersh, 2017). Careful examination of the mathematical knowing suggests a framework for organizing the categories of the material world, brain, mind, consciousness, and the self without reducing everything into matter, or equating consciousness with mind (as in Western philosophical traditions; Rao, 1998), or calling it an illusion, which is "the silliest view ever held in the history of human thought" (Strawson, 2018).

Knowing-within-mathematics involves abstracting concepts based on the measured properties of given mathematical objects, and interpreting the abstract theory to obtain concrete models (Lawvere, 1994a; Lawvere \& Rosebrugh 2003, pp. 154-155; Lawvere \& Schanuel 2009, pp. 135-136). For example, given a category of 
mathematical objects such as graphs, we measure their properties, say, dots and arrows. Comparing the measured properties of graphs gives rise to a theory of graphs (consisting of two graphs: dot and arrow, along with two graph morphisms specifying the inclusion of the dot into the arrow as source, target dot). In the light of this graph theory, a graph is modeled as a parallel pair of functions assigning to each arrow in the common domain set of arrows its source, target dot in the common codomain set of dots (Lawvere, 1994a, pp. 46-47; Lawvere \& Schanuel, 2009, pp. 149-151; Posina, Ghista \& Roy, 2017). Broadly speaking, mathematical generalization (theorizing and modeling) of a given category of particulars is determined by the choice of a mathematical doctrine (universal properties such as product; Lawvere, 1969; Lawvere, 2004, pp. 9-11). Abstract theories are calculated with respect to a doctrine; concrete models preserve the structure specified by the doctrine (Lawvere, 1994a; Lawvere, 2013b; Picado, 2007, pp. 14-15). Moreover, the processes of abstraction (to obtain theories) and interpretation (to obtain models) are adjoint functors (Lawvere, 2006). This pair of adjoint functors can be obtained as a "splitting" of a doctrine (or monad; also known as triple in Eilenberg \& Moore, 1965). Thus, the choice of a doctrine determines the way particulars (mathematical objects) are generalized (theorized and modeled). Subjective generals (theories and models) are about the given objective particulars (just as subjective appearances are appearances of the given objects), with the chosen mathematical doctrine serving as a subjective viewpoint determining how objects appear when viewed from that point of view.

Within the representational framework, conscious experience of a thing is a representation or a model of the thing (Chalmers, 2006). Based on the mathematical experience, a model of a thing is an appearance of the thing as seen in the light of a theory; so a model of an object depends not only on the object but also on the theory used to model. Comparing mental concepts to theories, we expect conscious experience (model) of a given stimulus to depend on mental concepts (theory). Consistent with this, mental concepts do determine the interpretation (conscious perception) of physical stimuli. What one sees, when one looks at any given image, depends on the mental concepts used to interpret the image. When looking at, say, R. C. James's image, one initially sees black and 84 
white blobs of various sizes and shapes. As soon as one brings the concept DALMATIAN to bear on the image, the same image is perceptually interpreted as a dog (Miller, 1999). In other words, our subjective experiences [of a situation] are models [of the situation] in the light of a theory (mental concept). Further examination of the mathematical experience shows that a mathematical theory of a category of mathematical objects depends not only on the objects but also on a mathematical doctrine, which can be thought of as a viewpoint. Changing the doctrine changes the way things are conceptualized and modeled (Lawvere, 1994a). In this context it may be noted that 'the doctrine can be varied, or "screwed up or down" as James Clerk Maxwell put it, in order to see various phenomena' (Lawvere, 2001). In light of this analogy of collective mathematical knowing, we suggest that the self, like a mathematical doctrine or monad (Lawvere, 2013b), determines all the thinking (mind / totality of theories) and experiencing (consciousness / totality of models) involved in individual knowing (Posina, Ghista \& Roy, 2017). Note that measured properties, abstract theories, and concrete models are all about mathematical objects, albeit from the perspective of a mathematical doctrine (monad). Along similar lines, neural coding in the brain, concepts in the mind, and conscious experiences are all about objective things, albeit from the perspective of an experiencing subject. In comparing mathematical objects, measured properties, abstract theories, concrete models, and the mathematical doctrine (constituting the collective knowing of mathematics) with physical stimuli, neural codes, mental concepts, conscious percepts, and the self (constituting the individual knowing of an experiencing subject), respectively, we find that the self can be thought of as a doctrine (or monad) within which all the mental conception and conscious perception takes place via neural measures of the properties of given physical stimuli. Simply put, there is more to the subjective appearance (model) of a particular object than all that is given in the object; we traced the "more" that is in the subjective appearance to the subject (doctrine). In this framework, the material world is conceptualized as a totality of all possible categories of objects (or a category of categories; Lawvere, 1966), while the brain is conceptualized as a totality of measured properties (or a category of functors; Lawvere \& Schanuel, 2009, 
pp. 369-370). With properties as functors and concepts as relations between properties (or natural transformations), mind, the totality of concepts or theories, is a "higher-dimensional" object compared to the brain. With percept as an interpretation (or a functor from the category objectifying a concept) into a background intuition, consciousness, the totality of perceptual experiences, is a further higher-dimensional object (Posina, Ghista \& Roy, 2017). In light of the conceptualization of the knowing self as a doctrine (monad), and in view of a doctrine splitting into the adjoint functors of abstraction and interpretation constituting mathematical knowing (as alluded to earlier), mind (totality of thinking) and consciousness (totality of experiencing; Koch, 2018) constituting knowing can be thought of as a "splitting" of the self. Stated differently, the knowing self (doctrine/monad) is the basic construct underlying the mental conception and conscious perception (generalization) of objective things (particulars).

\section{The Hardest Problem: The Society of Cognitive Selves}

The biological aspect of a society can be modeled as a set $S$ equipped with two functions, mother, father: $\mathrm{S} \rightarrow \mathrm{S}$, assigning to each individual in the society its parents, who are also individuals in the society S (Lawvere, 1999). Cultural aspects such as clan, marriage, and inheritance customs can be modeled by way of enriching the biological structure with clan and gender as labeling sets (Lawvere \& Schanuel, 2009, pp. 162-163). Here, we model the cognitive dimension of a society or the society of cognitive selves as a category of monads, with each monad as a cognitive self [in a cognitive society] determining how it experiences (thinks and perceives) the world. A cognitive self in a society, by virtue of being an object of a category, is completely determined by its relations to all cognitive selves in the society (cf. Yoneda lemma; Lawvere \& Rosebrugh, 2003, p. 249; for a detailed exposition of Yoneda lemma see Appendix A1 in Posina \& Roy, 2018). Thus, our model captures the relational nature of the self within society, as expressed by the Swahili word, ubuntu, which means, in the words of Desmond Tutu, "You are human precisely owing to relationships: you are a relational being" (Humphrey, 2007, p. 754). Human Society is, in the words of St. Augustine, "a region of 
unlikeness", a symbolic space wherein everything is experienced as something (Posina, 2017).

What is the nature of the totality of cognitive selves? One, possibly very naïve, answer would be: the totality of cognitive selves form a society (or at least the cognitive dimension of society). With cognitive selves as monads, society-the totality of cognitive selves - is the category of monads; more specifically, the category of composites of structure-semantics adjoint functors. What is the essence in which every cognitive self in the society (of Homo sapiens) partakes by virtue of being an object of the category of structure-semantics monads? Every cognitive self (object) of the (category of structure-semantics mondas i.e.) human society, perceives a particular as an exemplar of a general. A thorough characterization of the nature of human society, when modeled as the category of structure-semantics monads, requires a deeper study of the category, which is beyond the scope of the present paper, which, in turn, is to introduce the categorical method to cognitive and social scientists.

\section{Conclusion}

In the beginning, according to the eminently sensible scientific world-view, there was matter. Upon fast-forwarding to the present, we find, in addition to the world of material things, brains measuring these things, minds conceptualizing these measured things, and conscious experiences resulting from interpretations of these mental concepts, the experiencing subjects as the loci of all this thinking, seeing, and knowing. It is natural, given this temporal ordering with matter predating mind, consciousness, and the self, to wonder how mind or consciousness emerged from matter. Unfortunately, to put in the words of Fodor: "Nobody has the slightest idea how anything material could be conscious. Nobody even knows what it would be like to have the slightest idea about how anything material could be conscious" (Fodor, 1992, p. 5). Reminiscent of treating 'absence of evidence' as 'evidence of absence', many resorted to explaining away this scientific shortcoming of the materialist worldview by labeling conscious experiences and the experiencing self as illusions (Dennett, 1992; Metzinger, 2009; see also Strawson, 2018). Here we 
put forward mathematics as a thinking-device to help appreciate the reality of the self. The representational character of subjective experiences provides an immediate impetus for drawing parallels to mathematics. In comparing measured properties, abstract theories, background, concrete models, and doctrines (of mathematical knowing) to neural coding, mental concepts, intuition, conscious percepts, and experiencing subjects (of ordinary cognition), respectively, we find that the self is a necessary condition for the possibility of thinking and perceiving involved in experiencing in general and knowing in particular. Further investigations of the functional dependence of subjective generals (theories and models) on both objective particulars and the mathematical doctrine (monad) can help refine our understanding of the functional dependence of subjective experiences on both objective things and the knowing self.

Our functorial semantics of cognition framework (Lawvere, 1994a; Lawvere, 2004), enables the solution of mathematical problems analogous to a number of fundamental questions in consciousness studies. For example:

1. Formally, what are the conditions under which ' $C(R 1)$ is isomorphic to $C(R 2)$ ' implies ' $R 1$ is isomorphic to $R 2$ ', where $R 1$ and $R 2$ are elements of reality, while $C(R 1)$ and $C(R 2)$ are the corresponding conscious experiences, respectively.

2. Given that the theories of objects R1, R2 (of the category R objectifying reality) are $\mathrm{T}(\mathrm{R} 1), \mathrm{T}(\mathrm{R} 2)$, respectively, does $\mathrm{T}(\mathrm{R} 1+\mathrm{R} 2)$ $=\mathrm{T}(\mathrm{R} 1)+\mathrm{T}(\mathrm{R} 2)$ ? Expressing in words, is the theory of a sum of objects same as the sum of the theories of the objects? Is the process of theorizing (mental conception) respectful of the putting together of objects of reality (cf. DOG + CAT $=$ ANIMAL).

3. Given that the models of objects $\mathrm{R} 1$ and $\mathrm{R} 2$ (of reality $\mathrm{R}$ ) are $M(R 1)$ and $M(R 2)$, respectively, does $M(R 1 \times R 2)=M(R 1) \times M(R 2)$ ? In words, is the model of a product of objects same as the product of the models of the objects? For instance, in case of the category of graphs, the functor modeling graphs as parallel pairs of functions preserves the product operation (Lawvere \& Schanuel, 2009, pp. 141, 150, 258-260). Here we are asking a more general question: is the process of modeling (conscious perception) respectful of the 
many ways of putting together objects $(\mathrm{cf} . \mathrm{RED} \times \mathrm{SQUARE}=\mathrm{RED}$ SQUARE).

4. In a similar vein, what is the relation between products of models and sums of theories in context of the familiar exponential law, i.e. is $\mathrm{B}^{\mathrm{T}} \times \mathrm{B}^{\mathrm{V}}=\mathrm{B}^{(\mathrm{T}+\mathrm{V})}$, where $\mathrm{T}$ and $\mathrm{V}$ are theories (mental concepts), while $B$ is background category (cf. intuition) for [categories of] models (conscious percepts) $\mathrm{B}^{\mathrm{T}}, \mathrm{B}^{\mathrm{V}}$, and $\mathrm{B}^{(\mathrm{T}+\mathrm{V})}$ ? Answers to these mathematical questions can inform the grand philosophical problem of the comprehensibility of reality (Einstein, 1936), especially given our finite windows (concepts, percepts) on the world.

Summing it all, we identified representation as the basic notion of cognitive sciences. We then applied the mathematics of representation-functorial semantics-to model the society of cognitive selves. We hope our idealization of

Physical reality - Biological Brain - Mind - Intuition Consciousness - Self - Society

As

Particulars - Properties - Theories - Background - Models Monad - Category of Monads

will inspire cognitive and social scientists to bring functorial semantics in particular and category theory in general to bear on cognitive and social sciences.

\section{References}

Campos, M. V., \& Gutiérrez, A. M. L. (2015). The notion of point of view. In M. V. Campos and A. M. L. Gutiérrez (Eds.) Temporal Points of view: subjective and objective aspects, 1-57. Switzerland: Springer International Publishing.

Chalmers, D. J. (1995). Facing up to the problem of consciousness. Journal of Consciousness Studies, 2, 200-219.

Chalmers, D. J. (2006). The representational character of experience. In B. Leiter (Ed.) The future for philosophy, 153-181, New York, NY: Oxford University Press.

Dennett, D. (1992). The self as the center of narrative gravity. In F. Kessel, P. Cole, and D. Johnson (Eds.) Self and consciousness: multiple perspectives, 103-115, Hillsdale: Erlbaum. 
Eilenberg, S., and Moore, J. C. (1965). Adjoint functors and triples. Illinois Journal of Mathematics, 9, 381-398.

Einstein, A. (1936). Physics and reality. Journal of the Franklin Institute, 221, 349-382.

Fodor, J. (1992). The big idea: Can there be a science of the mind? Times Literary Supplement, 3 July, 5-7.

Gallagher, S. (2000). Philosophical conceptions of the self: Implications for cognitive science. Trends in Cognitive Sciences, 4, 14-21.

Gallagher, S. (2011). The Oxford handbook of the self. New York, NY: Oxford University Press.

Hamilton, A. (2015). Metaphysics and the anti-metaphysics of the self. Journal of Consciousness Studies, 22, 60-83.

Hersh, R. (2017). Mathematics as an empirical phenomenon, subject to modeling. Journal of Indian Council of Philosophical Research, 34, 331-342.

Hume, D. (1978). A treatise of human nature. Oxford: Oxford University Press.

Humphrey, N. (2007). The society of selves. Phil. Trans. R. Soc. B 362, 745754.

Koch, C. (2018). What is consciousness? Nature, 557, S9-S12.

Lawvere, F. W. (1963). Functorial semantics of algebraic theories. Proc. Natl. Acad. Sci. USA 50, 869-872.

Lawvere, F. W. (1966). The category of categories as a foundation for mathematics. In S. Eilenberg, D. K. Harrison, S. MacLane, and H. Rohrl (Eds.) Proceedings of the conference on categorical algebra, 1-20, New York, NY: Springer-Verlag.

Lawvere, F. W. (1969). Ordinal sums and equational doctrines. In B. Eckmann (Ed.) Seminar on triples and categorical homology theory, 141155. Berlin: Springer-Verlag.

Lawvere, F. W. (1980). Toward the description in a smooth topos of the dynamically possible motions and deformations of a continuous body. Cahiers de Topologie et Géométrie Différentielle Catégorique. 21, 337-392.

Lawvere, F. W. (1991). Some thoughts on the future of category theory. In A. Carboni, M. C. Pedicchio, and G. Rosolini (Eds.), Category theory, 279-281, New York, NY: Springer-Verlag.

Lawvere, F. W. (1992). Categories of space and of quantity. In J. Echeverria, A. Ibarra, and T. Mormann (Eds.) The Space of mathematics: philosophical, epistemological and historical explorations, 14-30. Berlin: DeGruyter.

Lawvere, F. W. (1994a). Tools for the advancement of objective logic: Closed categories and toposes. In J. Macnamara, and G. E. Reyes (Eds.) The Logical foundations of cognition, 43-56. New York, NY: Oxford University Press. 
Lawvere, F. W. (1994b). Cohesive toposes and Cantor's "lauter Einsen". Philosophia Mathematica, 2, 5-15.

Lawvere, F. W. (1999). Kinship and mathematical categories. In P. Bloom, R. Jackendoff, and K. Wynn (Eds.) Language, logic, and concepts, 411425. Cambridge, MA: MIT Press.

Lawvere, F. W. (2001). Sketches and Platonic ideas. Retrieved from https://facultypages.ecc.edu/alsani/ct01(9-12)/msg00116.html, Accessed 06 June 2019.

Lawvere, F. W. (2004). Functorial semantics of algebraic theories and some algebraic problems in the context of functorial semantics of algebraic theories. Reprints in theory and applications of categories, 5, 1-121.

Lawvere, F. W. (2006). Adjointness in foundations. Reprints in Theory and Applications of Categories, 16, 1-16.

Lawvere, F. W. (2013a). What are foundations of geometry and algebra? Celebrating Bill Lawvere and Fifty Years of Functorial Semantics. Retrieved from http:// www.math.union.edu/ niefiels/ 13conference/ Web/ Abstracts/Lawvere.pdf, Accessed 07 May 2015.

Lawvere, F. W. (2013b). Fifty years of functorial semantics. Celebrating Bill Lawvere and Fifty Years of Functorial Semantics. Retrieved from http:/ / www.math.union.edu/ niefiels/13conference/Web/Slides/ Fifty_ Years_of_Functorial_Semantics.pdf, Accessed 07 May 2015.

Lawvere, F. W., \& Rosebrugh, R. (2003). Sets for mathematics. Cambridge: Cambridge University Press.

Lawvere, F. W., \& Schanuel, S. H. (2009). Conceptual mathematics: A first introduction to categories. Cambridge: Cambridge University Press.

Menon, S. (2014). Brain, self and consciousness: explaining the conspiracy of experience. New Delhi: Springer India.

Metzinger, T. (2009). The ego tunnel: the science of the mind and the myth of the self. New York: Basic Books.

Midgley, M. (2014). Are you an illusion? New York: Routledge.

Miller, E. K. (1999). Straight from the top. Nature, 401, 650-651.

Picado, J. (2007). An interview with F. William Lawvere. CIM Bulletin, 23, 23-28.

Posina, V. R. (2017). Symbolic conscious experience. Tattva - Journal of Philosophy, 9, 1-12.

Posina, V. R. and Roy, S. (2018). Category theory and the ontology of sunyata. In Monograph on zero, (Netherlands: Brill Academic Publishers [forthcoming]).

Posina, V. R., Ghista, D. N., \& Roy, S. (2017). Functorial semantics for the advancement of the science of cognition. Mind $\mathcal{E}$ Matter, 15, 161-184.

Praetorius, N. (2016). Editorial introduction. Journal of Consciousness Studies, 23, 8-13. 
Rao, K. R. (1998). Two faces of consciousness: A look at Eastern and Western perspectives. Journal of Consciousness Studies, 5, 309-327.

Sen, A. (1993). Positional objectivity. Philosophy and Public Affairs, 22, 126145.

Siderits, M., Thompson, E., \& Zahavi, D. (2011). Self, No Self?: Perspectives from Analytical, Phenomenological, and Indian Traditions. Oxford: Oxford University Press.

Strawson, G. (2009). Selves: An essay in revisionary metaphysics. Oxford: Oxford University Press.

Strawson, G. (2018). The consciousness deniers. The New York review of books. Retrieved from https://www.nybooks.com/ daily/ 2018/ 03/ 13/the-consciousness-deniers/, Accessed 02 January 2020. 